\title{
Ocorrência de Dioctophyma renale em Galictis cuja ${ }^{1}$
}

\author{
Marivone V. Zabott ${ }^{2 *}$, Simone B. Pinto ${ }^{2}$, Aline M. Viott ${ }^{3}$, Raimundo A. Tostes ${ }^{3}$, \\ Laura H.F.B. Bittencourt ${ }^{4}$, Aline L. Konell ${ }^{5}$ e Leonardo Gruchouskei ${ }^{6}$
}

\begin{abstract}
Zabott M.V., Pinto S.B., Viott A.M., Tostes R.A., Bittencourt L.H.F.B., Konell A.L. \& Gruchouskei L. 2012. [Occurrence of Dioctophyma renale in Galictis cuja.] Ocorrência de Dioctophyma renale em Galictis cuja. Pesquisa Veterinária Brasileira 32(8):786-788. Laboratório de Parasitologia Veterinária, Universidade Federal do Paraná, Campus Palotina, Rua Pioneiro 2153, Palotina, PR 85950-00, Brazil. E-mail: marvalentim@ufpr.br

Dioctophymosis is a parasitic disease caused by Dioctophyma renale (Goeze, 1782) with a worldwide occurrence and affects domestic animals as well as wildlife. In March 2010, a ferret adult male, Galictis cuja (Molina, 1782), found dead by trampling in the county of Guaíra, state of Paraná, Brazil, was necropsied in the Veterinary Pathology Laboratory at Campus Palotina, Federal University of Paraná. The animal was in good nutritional condition and moderate autolysis. Three specimens of parasites were found in the abdominal cavity, but the kidneys were preserved. The parasites were fixed in acetic formaldehyde and sent to the Veterinary Laboratory of Parasitology, Campus Palotina, for identification. The parasites were identified as Dioctophyma renale, two females, one a $39 \mathrm{~cm}$ long and $4 \mathrm{~mm}$ wide and the other $16 \mathrm{~cm}$ long and $4 \mathrm{~mm}$ wide, and a male $16 \mathrm{~cm}$ long and $3 \mathrm{~mm}$ wide. This paper reports D. renale parasitism in G. cuja in the western of Paraná state, Brazil.
\end{abstract}

INDEX TERMS: Mustelidae, Galictis cuja, Dioctophyma renale, ferret.

RESUMO.- A dioctofimose é uma parasitose causada pelo Dioctophyma renale (Goeze, 1782) de ocorrência mundial e acomete animais domésticos e silvestres. Em março de 2010, um exemplar adulto (macho) de Galictis cuja (Molina, 1782), encontrado morto por atropelamento no município de Guaíra, Paraná foi encaminhado ao laboratório de Patologia Veterinária de Universidade Federal do Paraná (UFPR), Campus Palotina, para a realização da necropsia. 0 cadáver apresentava bom estado nutricional e autólise moderada. Foram observados três exemplares de parasitos na cavidade abdominal, mas os rins encontravam-se preservados. Os parasitos foram fixados em formol acético

\footnotetext{
${ }^{1}$ Recebido em 19 de julho de 2011.

Aceito para publicação em 25 de abril de 2012.

${ }^{2}$ Laboratório de Parasitologia Veterinária, Universidade Federal do Paraná (UFPR), Campus Palotina, Rua Pioneiro 2153, Palotina, PR 85950000, Brasil. *Autor para correspondência: marvalentim@ufpr.com.br

${ }^{3}$ Laboratório de Patologia Veterinária, UFPR-Campus Palotina, Palotina, PR.

${ }^{4}$ Laboratório de Parasitologia, Faculdade Assis Gurgacz (FAG), Cascavel, PR

${ }^{5}$ Graduanda do Curso de Medicina Veterinária, UFPR-Campus Palotina, Palotina, PR

${ }^{6}$ Residente do Curso de Medicina Veterinária, UFPR-Campus Palotina, Palotina, PR.
}

e encaminhados ao laboratório de Parasitologia Veterinária da UFPR para identificação. Os exemplares coletados foram identificados como Dioctophyma renale, sendo duas fêmeas, uma com $39 \mathrm{~cm}$ de comprimento por $4 \mathrm{~mm}$ de largura e a outra com $16 \mathrm{~cm}$ de comprimento por $4 \mathrm{~mm}$ de largura e, um macho com $16 \mathrm{~cm}$ de comprimento por $3 \mathrm{~mm}$ de largura. 0 presente trabalho relata a ocorrência de parasitismo por D. renale em G. cuja na região oeste do estado do Paraná.

TERMOS DE INDEXAÇÃO: Mustelidae, Galictis cuja, Dioctophyma renale, furão.

\section{INTRODUÇÃO}

Galictis cuja (Molina, 1782) é membro da ordem Carnivora, subordem Caniformia, família Mustelidae, subfamília Mustelinae, tribo Galictini. Na América do Sul a espécie é encontrada no sul do Peru, oeste da Bolívia, na região central do Chile, do Paraguai, do Uruguai e da Argentina e no leste e sudeste do Brasil (Yensen \& Tarifa 2003). Rápidos e ágeis, são bons nadadores e escaladores, sendo ativos à noite e de dia. Os mustelídeos possuem hábitos carnívoros e algumas espécies como o G. cuja são onívoras. Entre os furões, a dieta inclui pequenos mamíferos (roedores), aves e seus ovos, vertebrados ectotérmicos (lagartos, rãs e sapos), 
invertebrados e frutas (Yensen \& Tarifa 2003, Javorouski \& Passerino 2006). G. cuja apresenta o corpo alongado, cabeça pequena e ovalada, orelhas curtas e arredondadas, tórax estreito e uma cauda curta. Os membros locomotores são curtos e com cinco dedos. Outra característica é a presença de 20 a 21 vértebras na cauda dos adultos (Yensen \& Tarifa 2003).

A dioctofimose é uma parasitose causada por Dioctophyma renale (Goeze, 1782) de ocorrência mundial. 0 parasito tende a se localizar principalmente nos rins, ou livre na cavidade abdominal dos hospedeiros causando destruição progressiva das camadas cortical e medular dos rins, reduzindo os órgãos a uma cápsula fibrosa (Barros et al. 1990, Leite et al. 2005). 0 macho apresenta bolsa copuladora (desprovida de raios) localizada na extremidade posterior, no centro da bolsa abre-se o orifício cloacal de onde emerge um único espículo. A fêmea apresenta vulva simples na extremidade caudal obtusa e seus ovos são elípticos, castanhos e de casca espessa. Conhecido como estrôngilo gigante, o macho de $D$. renale pode atingir $45 \mathrm{~cm}$ de comprimento por $4 \mathrm{a} 6 \mathrm{~mm}$ da largura e a fêmea $100 \mathrm{~cm}$ de comprimento por $12 \mathrm{~mm}$ de largura (Alves et al. 2007, Amaral et al. 2008; Milanelo et al. 2009).

0 parasito já foi relatado em um grande número de animais silvestres incluindo martas (Mustela vison), doninhas (Mustela sibirica coreana), graxains (Cerdocyon thous), quatis (Nasua nasua), lontras (Lutra longicaudis), preguiças (Choloepus didactylus), lobos-guará (Chrysocyon brachyurus), coiotes (Canis latrans), lobos (Canis Lupus), raposas vermelhas (Canis vulpes), chacais (Canis mesomelas), rato-almiscarado (Ondatra zibethica), guaxinins (Procyon lotor), mão-pelada (Procyon cancrivorous), ursos (Ursus ursus) e recentemente foi detectado em um macaco-prego (Cebus apella) no estado do Pará, Brasil (Leite et al. 2005, Pesenti et al. 2007, Amaral et al. 2008, Ishizaki et al. 2010, Tokiwa et al. 2011). Adicionalmente existem relatos esporádicos de sua ocorrência em bovinos, equinos, suínos, felinos, focas e humanos todos considerados hospedeiros atípicos (Tokiwa et al. 2011). No Brasil existem relatos da ocorrência de parasitismo por $D$. renale, em furões na região litorânea do estado do Paraná (Barros et al. 1990) e no estado do Rio Grande do Sul no município de Pelotas (Pesenti et al. 2007). 0 objetivo desse trabalho é relatar a ocorrência de $D$. renale em $G$. cuja na região oeste do estado do Paraná.

\section{MATERIAL E MÉTODOS}

Em março de 2010 foi encontrado morto por atropelamento (licença do IBAMA número 21451-2) um exemplar macho de Galictis cuja, pesando $1,9 \mathrm{~kg}$ e medindo $59 \mathrm{~cm}$, no município de Guaíra, PR (latitude 2404'48” e longitude 54ํ1'21"). 0 mesmo foi encaminhado ao laboratório de Patologia Veterinária da UFPR, Campus Palotina para a realização da necropsia. Amostras teciduais foram coletadas, fixadas em formol tamponado a $10 \%$ e processadas rotineiramente pela coloração de hematoxilina-eosina para análise histopatológica. Os parasitos encontrados foram fixados em formol acético e submetidos para a identificação no laboratório de Parasitologia Veterinária da UFPR, Campus Palotina. Os parasitos foram identificados segundo Fortes (1997).

\section{RESULTADOS}

O cadáver apresentava bom estado nutricional e a autólise era moderada. Foram encontrados três exemplares de parasitos dispostos na cavidade abdominal, um deles havia perfurado o diafragma e estava parcialmente alojado na cavidade torácica. Os parasitos observados durante a necropsia foram identificados como Dioctophyma renale, sendo dois exemplares fêmeas (uma com $39 \mathrm{~cm}$ de comprimento por $4 \mathrm{~mm}$ de largura e a outra com $16 \mathrm{~cm}$ de comprimento por $4 \mathrm{~mm}$ de largura) e um exemplar macho (com $16 \mathrm{~cm}$ de comprimento por $3 \mathrm{~mm}$ de largura).

Não foram observadas alterações macroscópicas e microscópicas significativas. A análise de alguns órgãos do sistema digestório ficou prejudicada devido à autólise.

\section{DISCUSSÃO}

O parasitismo por Dioctophyma renale ocorre mais frequentemente no rim direito dos hospedeiros definitivos, onde as fêmeas, quando fecundadas, produzem ovos que são eliminados pela urina (Amaral et al. 2008). No presente estudo os parasitos foram encontrados livres na cavidade abdominal e torácica, sem nenhum envolvimento do parênquima renal, diferentemente do que foi observado por Barros et al. (1990) e Pesenti et al. (2007) que relataram o envolvimento do rim direito. A presença do parasito na cavidade torácica com consequente lesão de diafragma só tinha sido observada até o momento em cães, sendo associada a sintomatologia clínica de pneumotórax (Pesenti et al. 2007).

O local onde a larva infectante penetra no trato digestório do hospedeiro é determinante para a localização do parasito adulto. Larvas infectantes que penetram na curvatura menor do estômago evoluem para a forma adulta entre os lobos hepáticos o que explica a forma livre do parasito nas cavidades corpóreas. Já aquelas que migram pela curvatura maior do estômago e duodeno, tendem a se alojar no rim esquerdo e direito respectivamente. Localizações ectópicas de parasitos adultos já foram descritas no escroto, glândula mamária e tecido subcutâneo inguinal (Pesenti et al. 2007, Ishizaki et al. 2010).

D. renale são ovíparos e em condições adequadas no meio ambiente a primeira fase larval surge dentro dos ovos. Para prosseguir sua evolução, os ovos larvados devem ser ingeridos por hospedeiros intermediários. Oligoquetas de água doce são considerados os principais hospedeiros intermediários, crustáceos, peixes, sapos, rãs ou salamandras também são considerados intermediários no ciclo biológico do grande estrôngilo (Fortes 1997, Ishizaki et al. 2010, Tokiwa et al. 2011). Apesar do fato da fonte de infecção neste caso permanecer desconhecida, sabe-se que Galictis cuja alimentam-se frequentemente de sapos e rãs (Yensen \& Tarifa 2003).

\section{CONCLUSÃO}

Relata-se a ocorrência de parasitismo por Dioctophyma renale em Galictis cuja na região oeste do estado do Paraná. 
Agradecimentos.- Por sua colaboração na localização de material biológico para a realização deste trabalho, os autores agradecem ao Sr. Danilo Antônio Donin.

\section{REFERÊNCIAS}

Alves G.C., Silva D.T. \& Neves M.F. 2007. Dioctophyma renale: o parasita gigante do rim. Revta Cient. Eletron. Med. Vet. 4(8). Publicação Científica da Faculdade de Medicina Veterinária e Zootecnia de Graça/FAMED. Disponível em <http://revista.inf.br/veterinaria08/revisao/13.pdf> Acessado em 21 set. 2010.

Amaral L.C.D., Polizer K.A., Sant'Ana T.M. \& Neves M.F. 2008. Dioctophyma renale. Revta Cient. Eletron. Med. Vet. 4(10). Publicação Científica da Faculdade de Medicina Veterinária e Zootecnia de Graça/FAMED. Disponível em <http://revista.inf.br/veterinaria08/revisao/13.pdf> Acessado em 20 ago. 2010.

Barros D.M., Lorini M.L. \& Persson V.G. 1990. Dioctophymosis in the Little Grison (Galitis cuja). J. Wildl. Dis. 26(4):538-539.

Fortes E. 1997. Parasitologia Veterinária. 3ae ed. Editora Cone, São Paulo.

Ishizaki M.N., Imbeloni A.A., Muniz J.A.P.C., Scalercio S.R.R.A., Benigno R.N. M., Pereira W.L.A. \& Lacreta Junior A.C.C. 2010. Dioctophyma renale (Goeze, 1782) in the abdominal cavity of a capuchin monkey (Cebus apella), Brazil. Vet. Parasitol. 173: 340-343.
Javorouski M.L. \& Passerino A.S.M. 2007. Carnívora, Mustelidae (Ariranha, Lontra, Furão, Irara, Ferret), p. 547-570. In: Cubas Z.S., Silva J.C.R. \& Catão-Dias J.L. (Eds), Tratado de Animais Selvagens. Editora Roca, São Paulo.

Leite L.C., Círio S.M., Diniz J.M.F., Luz E., Navarro-Silva M.A., Silva A.W.C., Leite S.C., Zadorosnei A.C., Musiat K.C., Veronesi E.M. \& Pereira C.C. 2005. Lesões anatomopatológicas presentes na infecção por Dioctophyma renale (Goeze, 1782) em cães domésticos (Canis familiaris Linnaeus, 1758). Arch. Vet. Sci. 10(1):95-101.

Milanelo L., Moreira M.B., Fitorra L.S., Petri B.S.S., Alves M. \& Santos A.C. 2009. Occurrence of parasitism by Dioctophyma renale in ring-tailed coatis (Nasua nasua) of the Tiete Ecological Park, São Paulo, Brazil. Pesq. Vet. Bras. 29(12):959-962.

Pesenti T.C., Krüger C., Mascarenhas C.S. \& Müller G. 2007. Ocorrência de Dioctophyma renale (Goeze, 1782) em Galictis cuja no Rio Grande do Sul. XVI Congresso de Iniciação Científica UFPel, Pelotas.

Tokiwa T., Harunari T., Tanikawa T., Akao N. \& Ohta N. 2011. Dioctophyme renale (Nematoda: Dioctophymatoidea) in the abdominal cavity of Rattus norvegicus in Japan. Parasitol. Int. doi:10.1016/j.parint. 2011.03.003.

Yensen E. \& Tarifa T. 2003. Mammalian species, Galictis cuja. Am. Soc. Mammalogists. Lawrence, Kansas, 728:1-8. 